Background The outbreak of Coronavirus disease 2019 (COVID-19) caused by SARS-CoV-2 infection has become a global health emergency. We aim to decipher SARS-CoV-2 infected cell types, the consequent host immune response and their interplay in the lung of COVID-19 patients.

Methods We analyzed single-cell RNA sequencing (scRNA-seq) data of bronchoalveolar lavage fluid (BALF) samples from 10 healthy donors, 6 severe COVID-19 patients and 3 mild recovered patients. The expressions of SARS-CoV-2 receptors (ACE2 and TMPRSS2) were examined among different cell types. The immune cells infiltration patterns, their expression profiles, and interplays between immune cells and SARS-CoV2 target cells were further investigated.

Results Compared to healthy controls, ACE2 and TMPRSS2 expressions were significantly higher in lung epithelial cells of COVID-19 patients, in particular club and ciliated cells. SARS-CoV-2 activated pro-inflammatory genes and interferon/ cytokine signaling in these cells. In severe COVID-19 patients, significantly higher neutrophil, but lower macrophage in the lung was observed along with markedly increased cytokines expression compared with healthy controls and mild patients. By contrast, neutrophil and macrophage returned to normal level whilst more $\mathrm{T}$ and NK cells accumulation were observed in mild patients. Moreover, SARS-CoV-2 infection altered the community interplays of lung epithelial and immune cells: interactions between the club and immune cells were higher in COVID-19 patients compared to healthy donors; on the other hand, immuneimmune cells interactions appeared the strongest in mild patients.

Conclusions SARS-CoV-2 could infect lung epithelium, alter communication patterns between lung epithelial cells and immune system, and drive dysregulated host immune response in COVID-19 patients.

\section{IDDF2021-ABS-0210 ALTERED GUT METABOLITES AND BACTERIAL INTERACTIONS ARE IMPLICATED IN COLORECTAL CARCINOGENESIS AND CAN DETECT PRECANCEROUS AND CANCEROUS LESIONS}

\begin{abstract}
1,2Olabisi Oluwabukola Coker**, ${ }^{1,2}$ Changan Liu*, 1,3William Ka Kei Wu, 1,2 Sunny Hei Wong, ${ }^{4}$ Wei Jia, $1,2,5$ Joseph JY Sung, ${ }^{1,2}$ Jun Yu. ${ }^{1}$ State Key Laboratory of Digestive Disease, Li Ka Shing Institute of Health Sciences, CUHK Shenzhen Research Institute, The Chinese University of Hong Kong, Hong Kong, China; ${ }^{2}$ Department of Medicine and Therapeutics, The Chinese University of Hong Kong, Hong Kong, China; ${ }^{3}$ Department of Anaesthesia and Intensive Care, The Chinese University of Hong Kong, Hong Kong, China; ${ }^{4}$ School of Chinese Medicine, Hong Kong Baptist University, Kowloon Tong, Hong Kong, China; ${ }^{5}$ Lee Kong Chian School of Medicine, Nanyang Technology University, Singapore
\end{abstract}

\subsection{6/gutjin-2021-IDDF.6}

Background Gut microbiota contributes to colorectal cancer (CRC) pathogenesis through specific pathogens and their metabolites. We aim to determine gut microbiota-associated metabolites and their linkage to CRC.

Methods We performed metabolomics by gas chromatography coupled to time-of-flight mass spectrometry, and shotgun metagenomics analysis on fecal samples from 386 subjects [118 CRC patients, 140 colorectal adenomas (CRA) patients and 128 normal controls (NC)]. Stepwise logistic regression models were used to identify metabolites and bacterial markers discriminating CRC stages. Associations among CRC-associated metabolites and bacteria were estimated with zero-inflated negative binomial regressions analysis.

Results Principal component analysis and partial least squaresdiscriminant analysis showed differences in the gut metabolite profiles among CRC, CRA and NC groups. Norvaline and myristic acid showed increasing trends from NC, through CRA, to CRC. CRC-associated metabolites were enriched in branched-chain amino acids and aminoacyl-tRNA biosynthesis pathways. Twenty metabolites classified CRC from NC subjects with an area under the curve (AUC) of 0.80, and CRC from CRA with AUC of 0.79 . Combinations of metabolites and bacterial markers improved the diagnostic performances (CRC vs NC, AUC: 0.94; CRC vs CRA, AUC 0.92; CRA vs NC, AUC: 0.86), indicating a potential for early diagnosis of colorectal neoplasia. Moreover, relationships among CRC-associated metabolites and bacteria were altered across CRC stages; certain associations exhibited increasing or decreasing strengths while some were reversed from negative to positive or vice versa.

Conclusions Gut metabolites are altered in colorectal carcinogenesis. The combination of metabolites and bacterial species can increase the chance of a non-invasive diagnosis of colorectal neoplasia.

\section{Basic Hepatology}

\section{IDDF2021-ABS-0145 TURNING IMMUNOLOGICALLY COLD TUMORS INTO HOT ONES BY ACTIVATING HEPATOMA-INTRINSIC FADD/NF-KB/CCL5 PATHWAY}

Jiahuan Lu*, Jing Wang, Yalin Tu, Weiqin Yang, Wenshu Tang, Zhewen Xiong, Alfred SzeLok Cheng, Anthony Wing-Hung Chan, Ka-Fai To. Chinese University of Hongkong, Hong Kong

\subsection{6/gutjnl-2021-IDDF.7}

Background Lymphoepithelioma-like hepatocellular carcinoma (LEL-HCC) as a distinct variant of HCC displayed immunologically hot tumor features with prominent tumor-infiltrating $\mathrm{CD}^{+} \mathrm{T}$ lymphocytes (TILs). Our whole exosome sequencing data found an increased prevalence of chromosome 11q13.3 amplification in LEL-HCC, in which contains fas-associated death domain (FADD) that displayed a strongest positive correlation with differentially expressed genes function in TIL migration. We hence aim to elucidate the functional roles and molecular mechanisms of hepatomaintrinsic FADD in regulating TIL abundance in general HCC patients.

Methods FADD-overexpressed HepG2 (FADD-oe) and FADDknockdown huh7 cells (FADD-kd) were constructed to investigate cell growth rate by colony formation and MTS assay in vitro, subcutaneous tumor in immunodeficient nude and NOD/SCID mice in vivo. A co-culture model of human peripheral blood-derived- $\mathrm{CD}^{+} \mathrm{T}$ cells and HepG2-oe or Huh7-kd in vitro, $\mathrm{T}$ cell adoptive transfer and humanized mice models in vivo were used to detect $\mathrm{T}$ migration preference. The downstream functional chemokines and molecular pathways controlled by FADD were determined by RT-qPCR, ELISA, western blot as well as gene modulation assays. Finally, 
the associations among FADD (Fadd) expression, TIL proportion and patient prognosis or tumor development were evaluated by NanoString/immunohistochemistry analysis in HCC patients or RT-qPCR/flow cytometry in orthotopic HCC mouse models.

Results $\mathrm{CD}^{+} \mathrm{T}$ cell migration rate was significantly enhanced in FADD-oe tumors, but reduced in FADD-kd tumors in both HCC cells-T cell co-culture system in vitro and subcutaneous tumor models in vivo. Mechanistically, FADD activated NF- $\kappa$ $\mathrm{B}$, which in turn promoted $\mathrm{T}$ cell trafficking chemokine CCL5 production. Furthermore, Fadd expression was positively correlated with $\mathrm{Ccl} 5$ and TIL proportions, but negatively correlated with orthotopic HCC tumor weights. As we showed that FADD was positively correlated with $\mathrm{CD}^{+} \mathrm{T}$ cells in HCC patients, our data pinpointed that FADD controls TIL abundance in HCC.

Conclusions Our findings reveal an underlying mechanism of TIL accumulation in HCC, which provides a novel strategy of FADD activation in turning immunologically cold tumors into hot ones for better prognosis and immunotherapeutic responses.

\section{IDDF2021-ABS-0177 RNF135 PROMOTER METHYLATION IS ASSOCIATED WITH IMMUNE INFILTRATION AND PROGNOSIS IN HEPATOCELLULAR CARCINOMA}

Guijun Zhao*, Xiong Liang, Yu Bai, Hao Li, Guang Yong, Yusha Liu, Yukun Liang, Xiao Wang. Endoscopy Center, Inner Mongolia key laboratory of endoscopic digestive diseases, Inner Mongolia People's Hospital, Hohhot, Inner Mongolia Autonomous Region, China

\subsection{6/gutjnl-2021-IDDF.8}

Background RING finger protein 135 (RNF135) has an important role in the occurrence of many cancers, however, the regulation and function of RNF135 in hepatocellular carcinoma (HCC) remains unknown.

Methods The promoter methylation status and mRNA expression of RNF135 were evaluated by methylation-specific PCR and real-time PCR in HCC cell lines and tissues, and further analyzed from The Cancer Genome Atlas database. Transwell migration, wound healing assay, cell viability, and colony formation assay were performed to investigate the function of RNF135. GSEA analysis, TIMER database and ESTIMATE algorithm were used to decipher the associated pathway and immune infiltration. The survival analysis was applied to assess the prognostic value of RNF135.

Results RNF135 expression was downregulated in 5 of 8 HCC cell lines and HCC tissues, and was negatively correlated with its promoter hypermethylation. Demethylating regent decitabine restored RNF135 expression on the cellular level. Knockdown of RNF135 expression enhanced the migration of HCC cells, while RNF135 overexpression repressed cell migration. Bioinformatics analysis revealed a positive relationship between RNF135 expression and six immune cell infiltrates (B cells, CD4 + T cells, CD8 + T cells, neutrophils, macrophages, and dendritic cells). Survival analysis disclosed that RNF135 hypermethylation is independently associated with poor clinical outcomes in HCC.

Conclusions Decreased RNF135 expression driven by promoter hypermethylation is frequently occurred in HCC and associated with prognosis of HCC. RNF135 functions as a tumor suppressor and involves in tumor immune microenvironment in HCC.

\section{IDDF2021-ABS-0185 OVERCOME PRIMARY RESISTANCE TO IMMUNE CHECKPOINT BLOCKADE IN HEPATOCELLULAR CARCINOMA}

${ }^{1}$ Xiaoyu Liu*, ${ }^{1}$ Jingying Zhou, ${ }^{2}$ Man Liu, ${ }^{3}$ Ying Wang, ${ }^{3}$ Mengying Hu, ${ }^{3}$ Rihe Liu, ${ }^{3}$ Leaf Huang, ${ }^{1}$ Hanzhuang Liu, ${ }^{1}$ Alfred Sze-Lok Cheng. ' School of Biomedical Sciences, The Chinese University of Hong Kong, Hong Kong, China; ${ }^{2}$ The First Affiliated Hospital, Sun YatSen University, Guangzhou, China; ${ }^{3}$ Department of Biomedical Engineering, The Chinese University of Hong Kong, Hong Kong, China; ${ }^{4}$ Eshelman School of Pharmacy, University of North Carolina, Chapel Hill, NC, USA; ${ }^{5}$ Lee Kong Chian School of Medicine, Nanyang Technological University, Singapore; ${ }^{6}$ Institute of Precision Medicine, The First Affiliated Hospital, Sun Yat-sen University, Guangzhou, China

\subsection{6/gutjnl-2021-IDDF.9}

Background Although immune checkpoint blockade (ICB) such as anti-programmed cell death 1 or its ligand (anti-PD-1/PDL1) has revolutionized the treatment paradigm for cancer patients, most hepatocellular carcinoma (HCC) patients did not show benefits in the clinical scenario. The aim of this study is to investigate the underlying mechanisms of immunotherapy resistance in HCC and explore therapeutic approaches for enhancing HCC immunotherapy.

Methods Fibrosis-associated HCC mouse model was established by chemical induction and tumor inoculation. Non-alcoholic fatty liver disease (NAFLD)-associated HCC was established by high-fat high carbohydrate diet and tumor implantation. Integrated analysis of single-cell RNA sequencing and bulk RNA sequencing were applied to identify monocytic myeloid-derived suppressor cells (M-MDSC) signature genes. Immune profiles were determined by flow cytometry.

Results We found M-MDSC was specifically enriched in nonresponders and a protein phosphatase was identified as a novel signature gene in M-MDSC. Inhibition of this protein phosphatase suppressed M-MDSC expansion and reduced reactive oxygen species generation, which holds promise in ameliorating the immunosuppressive microenvironment for tumor eradication. To further improve HCC therapy in ICB manner, we found an elegant nanoparticle-parceled PD-L1 trap which was superior to the conventional antibody-mediated ICB therapy. This PD-L1 trap was more up taken by the fibrotic liver environment compared to the normal liver indicating it would be suitable for majority of HCC patients. Ultimately, the PDL1 trap triggered robust anti-tumor response in both fibrotic HCC and NAFLD-associated HCC. Mechanistically, PD-L1 trap exerted potent immune-editing function by diminishing immunosuppressive cells and boosting $\mathrm{CD} 8^{+} \mathrm{T}$ cells infiltration to the tumor. Those tumor-infiltrating $\mathrm{CD}^{+} \mathrm{T}$ cells were more cytotoxic with augmented cytokines production. More importantly, improved survival was observed in PD-L1 traptreated HCC.

Conclusions We uncovered a novel target for immunosuppressive myeloid cells which was closely associated with immunotherapy resistance in HCC. Moreover, a novel ICB approach showed translational potential for treating HCC with the enhanced therapeutic effect but reduced toxicity. We anticipate providing more efficacious options for HCC patients with long-term benefits. Acknowledgements: This study is supported by HMRF (16170451, 07180556) and $\mathrm{Li} \mathrm{Ka}$ Shing Foundation. 